\title{
Whole lesion histogram analysis of apparent diffusion coefficients on MRI predicts disease-free survival in locally advanced squamous cell cervical cancer after radical chemo-radiotherapy
}

\author{
Bo Zhao, Kun Cao ${ }^{*}$, Xiao-Ting Li, Hai-Tao Zhu and Ying-Shi Sun ${ }^{*}$
}

\begin{abstract}
Background: The aim was to investigate the prognostic value of MR apparent diffusion coefficients (ADC) using histogram analysis (HA) in predicting disease-free survival (DFS) of cervical cancer after chemo-radiation therapy.

Methods: We retrospectively analyzed 103 women with pathologically proven squamous cell uterine cancer who received chemo-radiation therapy between 2009 and 2013. All patients were followed up for more than 2 years. Pre-treatment MR images were retrieved and imported for HA using an in-house developed software program based on 3D Slicer. Regions of interest of whole tumors were drawn manually on DWI with reference to T2WI. HA features (mean, max, min, 50, 10, 90\%, kurtosis, and skewness) were extracted from apparent diffusion coefficient (ADC) maps and compared between the recurrence and non-recurrence groups after the 2-year follow-up. Univariate and multivariate Cox regression analysis was used to correlate ADC HA features and relevant clinical variables (age, grade, maximal diameter of tumor, FIGO stage, SCC-Ag) with DFS.

Results: One hundred three patients with stage IB-IV cervical cancers were followed up for 2.0-94.6 months (median 48.9 months). Twenty patients developed recurrence within 2 years. In the recurrence group, the min $(P=0.001)$ and $10 \%(P=0.048)$ ADC values were significantly lower than those of the non-recurrence group. Univariate and multivariate Cox regression analysis revealed that $\mathrm{ADC}_{\min }(P=0.006, \mathrm{HR}=0.110)$ was significantly correlated with DFS.

Conclusion: Pre-treatment volumetric $A D C_{\text {min }}$ in histogram analysis is an independent factor that is correlated with DFS in cervical cancer patients treated with chemo-radiation therapy.
\end{abstract}

Keywords: Uterine cervical neoplasm, Diffusion-weighted MRI, Survival

\section{Background}

Uterine cervical cancer is one of the most common malignancies in the female gynecologic system. Due to extremely unbalanced economical and healthcare levels across the world, the incidence and prognosis of cervical cancer vary dramatically among different countries and

\footnotetext{
*Correspondence: kun-cao@hotmail.com; sys27@163.com

${ }^{+}$Kun Cao and Ying-Shi Sun contributed equally to this work.

Department of Radiology, Key Laboratory of Carcinogenesis and Translational Research, Ministry of Education, Peking University Cancer Hospital and Institute, No. 52 Fucheng Rd, Haidian District, Beijing 100142, China
}

areas. Most cervical cancer patients in China are at advanced stages when diagnosed. According to the FIGO stage system and NCCN guidelines, locally advanced cervical carcinoma (LACC) usually refers to stage IB2 to IVA cancers, and concurrent chemoradiotherapy (CCRT) is suggested as the standard treatment strategy for such patients [1]. The current concept of cancer treatment calls for personalized treatment in order for the patient to benefit the most and to maximally increase survival time.

Among the factors that are related to the survival rate of cervical cancer, tumor size is the most well-accepted

(c) The Author(s). 2019 Open Access This article is distributed under the terms of the Creative Commons Attribution 4.0 International License (http://creativecommons.org/licenses/by/4.0/), which permits unrestricted use, distribution, and 
one; it has already been included as a key factor in the FIGO staging system. Other risk factors include positive lymph nodes, parametrial extension, positive surgical margins, lymph vascular space invasion, and depth of invasion [2], but most of this information can only be acquired by operational pathology, which is impossible for LACC patients subjected to radical radiation. Imaging has the ability to provide more information in vivo before treatment, so much research has been carried out on the selection of imaging parameters, such as enhancing patterns, to predict the survival rate of cervical cancer. Comparing with dynamic contrast enhanced parameters, DWI has obvious advantages, such as nonuse of contrast radium, relatively stable image quality, and a universally accepted quantitative index, the apparent diffusion coefficient (ADC).

The results regarding ADC values in predicting treatment effects vary greatly. Some studies showed low ADC values are related with recurrence and a poor survival rate $[3,4]$, while some found low ADC values in patients with good treatment responses $[5,6]$. Other studies concluded that the available evidence is insufficient to use pre-treatment $\mathrm{ADC}$ values to predict treatment efficacy [7-9]. One of the main disadvantages of ADC measurements is that the location and the sizes of regions of interests (ROIs) may affect the values, which is a common practical problem in almost all parameter measurements. Therefore, the application of histogram analysis (HA) is validated, which generates more indices to describe the distribution of each voxel within ROIs, instead of a single mean value. Studies on HA did achieve inspiring results in predicting cervical cancer survival rates, but large differences exist between studies. One of the possible reasons may still be the choice of ROIs, as some studies used maximum single slices instead of whole tumor slices. We suspected that whole lesion ROIs are able to reflect lesion characteristics to the greatest extent, while small ROIs or single slice ROIs all have the possibility of selection bias. Therefore, in this study, we use whole lesion ROIs and HA to assess the possibility to use ADC values to predict disease-free survival (DFS) of cervical cancer for a follow-up period of 5 years.

\section{Methods}

\section{Patients}

Informed consents were waived by the institutional Ethics Committee for this retrospective study. All patients with pathologically diagnosed squamous cell uterine cervical cancer in our institute from 2009 to 2013 were reviewed, and 152 consecutive patients were found to meet the following inclusion criteria: (a) the tumor was in stage IB to IVA, (b) the patient received concurrent chemoradiation in our radiation department, (c) pre-treatment pelvic MR was performed in our hospital and was within 30 days before treatment started, and (d) valid follow-up information could be acquired from the medical records. After retrieving MR images and clinical medical records, 49 patients were excluded, including 32 with sagittal instead of axial DWI, 8 with poor quality DWI images, 5 with a tumor with a long diameter of less than $1 \mathrm{~cm}, 1$ with small cell lung cancer, and 3 with a follow-up time of less than 2 years. We recorded the relevant clinical information, including age, grade, FIGO stage, and serum levels of squamous cell carcinoma antigen (SCC-Ag). In total, 103 subjects were enrolled in the study. The follow-up time was defined as from the date of first radiation to either the recurrence date or the last visit date with no events. The median follow-up time for the whole cohort was 48.9 months (range 2.0-94.6 months). Among them, 55 patients free of recurrence or metastasis at the end of the follow-up period (median follow-up time 59.7 months).

\section{Treatment and outcome evaluation}

All patients underwent standard radiotherapy in combination with concurrent cisplatin-containing chemotherapy. Radiotherapy consisted of external beam radiation (4050.4 Gy in $1.8-2 \mathrm{~Gy}$ daily fractions using $10-\mathrm{MV}$ photons) and intracavitary brachytherapy (30-35 Gy to point A by 5 Gy fractions in 3 weeks). Six courses of cisplatin-based chemotherapy $\left(40 \mathrm{mg} / \mathrm{m}^{2}\right.$ dosage) were delivered weekly at the same time with radiotherapy.

The primary outcome was DFS, defined as the period of time from the first radiation treatment to the date of developing any recurrence (local or distant relapse, metastasis). Patients with persistent disease were considered to have relapsed on the first day of completing radiation therapy. Recurrence data were taken from the medical records, with evidence based on the diagnosis by the treating physician and imaging (18F-fluorodeoxyglucose [18F-FDG] positron emission tomography/computed tomography [PET/CT], MRI, CT) or biopsy results. Recurrence group and non-recurrence group were divided based on DFS $\leq 2$ year and DFS $>2$ years.

\section{MR imaging}

All examinations were performed on 1.5-T MR scanners (GE optima 1.5 T MR360 and GE signa 1.5 T EchoSpeed Plus) with four-channel or eight-channel torso phasedarray body coil. The scanning range was set to cover the entire pelvis from the level of the anterior superior iliac spine to the inferior level of the symphysis pubis. All patients were required to fast at least $2 \mathrm{~h}$ before the examination. After excluding the contradictions, patients were asked to inject $5 \mathrm{ml}$ of anisodamine about $20-30 \mathrm{~min}$ before the examination to reduce bowel motion artifacts.

MR sequences included T1WI, T2WI, and DWI. The standard MR scan protocol was kept identical each time 
and was as follows: (a) axial T1WI: fast spin-echo (FSE) sequence, repetition time $(\mathrm{TR})=726 \mathrm{~ms}$, echo time $(\mathrm{TE})=12 \mathrm{~ms}$, matrix size $=288 \times 256$, field of view $(\mathrm{FOV})=26-36 \mathrm{~cm}$, slice thickness/intersection gap $=8 / 1$ $\mathrm{mm}$, number of excitations $(\mathrm{NEX})=1$; (b) axial T2WI: FSE-XL, TR $=5182 \mathrm{~ms}, \mathrm{TE}=80 \mathrm{~ms}$, matrix size $=352 \times$ $320, \mathrm{FOV}=26-30 \mathrm{~cm}$, slice thickness/intersection gap $=$ $8 / 1 \mathrm{~mm}, \mathrm{NEX}=2$; (c) sagittal T2WI: FSE-XL, TR $=4138$ $\mathrm{ms}, \mathrm{TE}=102 \mathrm{~ms}$, matrix size $=320 \times 288, \mathrm{FOV}=24-36$ $\mathrm{cm}$, slice thickness/intersection gap $=5 / 1 \mathrm{~mm}, \mathrm{NEX}=4$; (d) axial DWI: single-shot echo-planar imaging sequence $, \mathrm{TR}=3500 \mathrm{~ms}, \quad \mathrm{TE}=$ minimum time, matrix size $=128 \times 128, \quad F O V=36 \mathrm{~cm}$, slice thickness $/$ intersection gap $=8 / 1 \mathrm{~mm}, \mathrm{NEX}=4, \mathrm{~b}$-value $=0$ and $1000 \mathrm{~s} /$ $\mathrm{mm}^{2}$. All slice lines of DWI were copied from the axial T2WI to make sure the images were all at the same table position.

\section{Image analysis}

All images were retrieved from local PACS (Huahai, China). By putting axial T2WI and DWI images side by side and also referring to the sagittal T2WI, ROIs were drawn manually slice by slice on DWI images along the edge of the lesions in order to cover as much tumor area as possible without excluding cystic, hemorrhagic or necrotic areas (Fig. 1). ROIs were initially drawn by a junior radiology resident (Z.B.) with 3 years' experience in $M R$ reading and then reviewed and corrected by a senior attending radiologist (C.K.) with more than 10 years' experience in gynecologic imaging. Both radiologists were blinded to patients' outcomes during the data collection period. Whole lesion ROIs were thus achieved to acquire volumetric data of tumors.
Axial DWI images were loaded into a post-processing workstation (GE AW 4.6) and parametric ADC maps were generated automatically in the FUNCTOOL program. Parametric maps along with DWI and ROIs were all transferred to house-made radiomics software based on the 3D Slicer platform. ROIs were registered to the parametric maps. Histograms and corresponding ADC parameters values were automatically generated by the software. Excel software was used to sum up the parameters values of ROI at all slices of the tumor respectively and then calculate the average value. The HA program in the software was generated the following 8 parameters for ADC values: mean, median, maximum, minimum, 10th percentile, 90th percentile, kurtosis, and skewness. The maximal diameter of tumor (MDT) was calculated on two-dimensional axial ADC metrics.

\section{Statistical analyses}

Continuous variables are presented as mean \pm SD. Categorical variables are presented as counts and percentages. The clinical variables and ADC parameter values were compared between the recurrence and nonrecurrence groups using the Wilcoxon rank-sum test or the chi-square test. Univariate and multivariate Cox regression analyses were used to evaluate the potential prognostic value of ADC parameters and relevant clinical variables (i.e., age, MDT, grade, FIGO stage, SCC$\mathrm{Ag}$ ) for DFS. For the multivariate analysis, parameters were selected by using stepwise selection and by considering the following covariates with a $P$-value less than 0.3 in the univariate analysis. The receiver operating characteristic (ROC) curve was drawn to determine the cutoff value of the parameters using the maximum
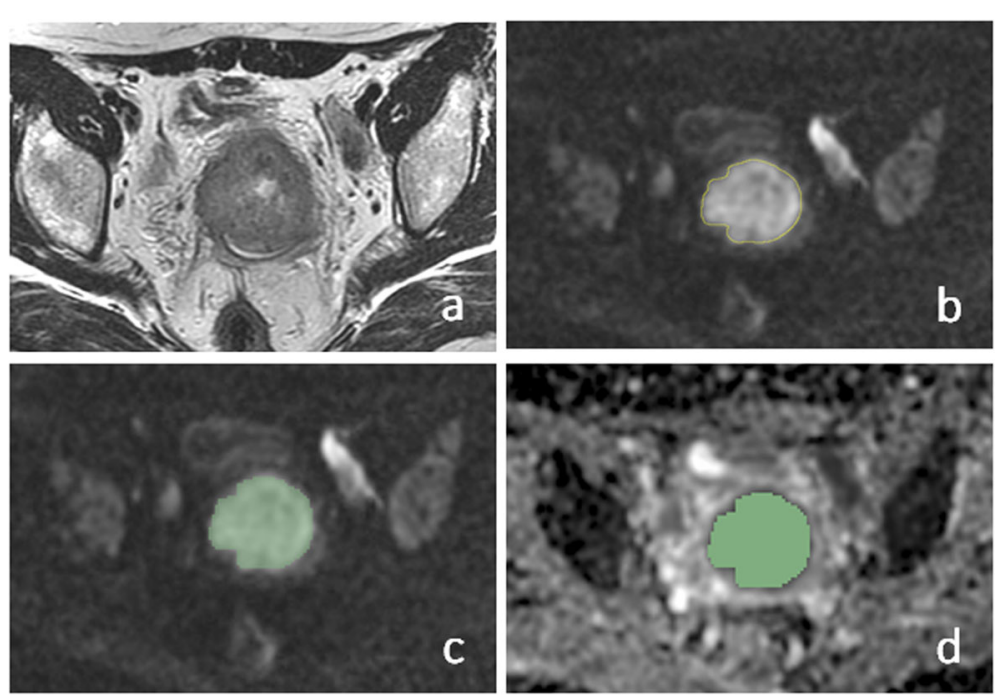

Fig. 1 Manual segmentation of ROls in cervical lesion and schematic diagram of parameters. a-c Referring to T2WI and DWI, ROls were drawn manually slice by slice on DWI images along the edge of the lesions in order to cover as much tumor area as possible without excluding cystic or necrotic areas. $\mathbf{d}$ The same ROls were registered to ADC maps 
Youden index. The Kaplan-Meier survival curve was drawn and log-rank estimates were obtained. All statistical analyses were performed using SPSS 22.0. All reported values are two-tailed, and $P$-values $<0.05$ were considered statistically significant.

\section{Results}

Clinical characteristics are listed in Table 1. All patients were followed up for more than 2 years, the median follow-up period was 59.7 months (range, 25.5-94.6 months) for surviving patients. Patient characteristics are listed in Table 1. Of the 103 women analyzed, tumor recurrence was observed in 29 cases $(28.2 \%)$ at the end of the follow-up period; 11 in the first year, 9 in the second year, and 9 patients suffered from recurrence after more than 2 years of follow-up. Of the 29 patients with tumor recurrence, 3 had persistent cervical cancer, 15 patients showed distant metastasis, and 11 patients showed local relapse in the pelvic cavity. The 1-year and 2 -year DFS rates were 89.3 and $80.5 \%$, respectively. Patients characteristics in the recurrence and nonrecurrence groups are presented in Table 2. Pretreatment $\mathrm{ADC}_{\text {min }}$ and $\mathrm{ADC}_{10 \%}$ were $0.588(0.163-5.351)$ $\left(\times 10^{-3} \mathrm{~mm}^{2} / \mathrm{s}\right)$ and $0.776(0.543-8.134)\left(\times 10^{-3} \mathrm{~mm}^{2} / \mathrm{s}\right)$ in the recurrence and non-recurrence groups, respectively.

The values of $\mathrm{ADC}_{\min }(P=0.001)$ and $\mathrm{ADC}_{10 \%}(P=$ 0.048 ) were significantly lower in the recurrence group than in the non-recurrence group. In the univariate analysis, age, $\mathrm{ADC}_{\min }, \mathrm{ADC}_{10 \%}$, and $\mathrm{ADC}_{\text {skewness }}$ were associated with DFS (Table 3). In the multivariate Cox analysis, $\mathrm{ADC}_{\min }$ of the tumor was the most significant

Table 1 Patient and Tumor characteristics

\begin{tabular}{ll}
\hline Characteristic & Value \\
\hline Patient number & 103 \\
Age (years) $^{a}$ & $51(27-78)$ \\
Histology & Squamous cell carcinoma \\
FIGO stage & \\
IB & $18(17.5 \%)$ \\
IIA/B & $16 / 48(15.5 \% / 46.6 \%)$ \\
IIIA/B & $6 / 13(5.9 \% / 12.6 \%)$ \\
IV & $2(1.9 \%)$ \\
Grade of differentiation & \\
Well/moderate & $59(57.3 \%)$ \\
Poor & $44(42.7 \%)$ \\
MDT (mm) & $45.1(14.8-90.6)$ \\
SCC-Ag (ng/ml) & $4.0(0.4-81.9)$ \\
Follow-up time (months) & $48.9(2.0-94.6)$
\end{tabular}

Note: Data are number and data in parentheses are percentages; ${ }^{2}$ Data are median and data in parentheses are rang; FIGO International Federation of Gynecology and Obstetrics, MDT Maximal diameter of the tumor, SCC-Ag Squamous cell carcinoma antigen
Table 2 Characteristics of patients and values of ADC histogram parameters in recurrence (DFS $\leq 2$ years) and non-recurrence (DFS $>2$ years) groups

\begin{tabular}{llll}
\hline Parameter & $\begin{array}{l}\text { Non-recurrence } \\
n=83(80.6 \%)\end{array}$ & $\begin{array}{l}\text { Recurrence } \\
n=20(19.4 \%)\end{array}$ & $P$-value \\
\hline Age (years) & $50.4 \pm 9.2$ & $51.3 \pm 11.4$ & 0.973 \\
Grade & & & \\
$\quad$ Well/moderate & $48(46.6 \%)$ & $11(10.7 \%)$ & 0.075 \\
$\quad$ Poor & $35(34.0 \%)$ & $9(8.7 \%)$ & \\
$\quad$ MTD & $42.9 \pm 16.0$ & $48.4 \pm 13.1$ & 0.115 \\
FIGO stage & & & 0.996 \\
$\quad$ IB/IIA & $29(28.2 \%)$ & $7(6.8 \%)$ & \\
IIB/III/IV & $54(52.4 \%)$ & $13(12.6 \%)$ & \\
SCC-Ag & $9.7 \pm 14.4$ & $11.3 \pm 15.2$ & 0.957 \\
Median $\left(\times 10^{-3} \mathrm{~mm}^{2} / \mathrm{s}\right)$ & $1.094 \pm 1.117$ & $0.912 \pm 0.537$ & 0.443 \\
Max $\left(\times 10^{-3} \mathrm{~mm}^{2} / \mathrm{s}\right)$ & $2.202 \pm 1.664$ & $2.144 \pm 0.328$ & 0.352 \\
Mean $\left(\times 10^{-3} \mathrm{~mm}^{2} / \mathrm{s}\right)$ & $1.056 \pm 0.156$ & $1.013 \pm 0.119$ & 0.372 \\
Min $\left(\times 10^{-3} \mathrm{~mm}^{2} / \mathrm{s}\right)$ & $0.648 \pm 0.570$ & $0.356 \pm 0.383$ & $0.001^{*}$ \\
10\% $\left(\times 10^{-3} \mathrm{~mm}^{2} / \mathrm{s}\right)$ & $0.900 \pm 0.817$ & $0.757 \pm 0.173$ & $0.048^{*}$ \\
$90 \%\left(\times 10^{-3} \mathrm{~mm}^{2} / \mathrm{s}\right)$ & $1.437 \pm 1.470$ & $1.305 \pm 0.219$ & 0.900 \\
Kurtosis & $5.575 \pm .254$ & $4.910 \pm 2.541$ & 0.286 \\
Skewness & $1.184 \pm 0.602$ & $0.912 \pm 0.537$ & 0.067 \\
\hline
\end{tabular}

Note: Data are presented as mean $\pm S D$, with Wilcoxon rank-sum test. Data are presented number (percentages), with chi-square test. ${ }^{*} P$-value $<0.05$

predictor for patient DFS $(P=0.006, \mathrm{HR}=0.110)$, where a higher $\mathrm{ADC}_{\text {min }}$ was significantly associated with poor DFS (Table 3).

ROC analysis of tumor recurrence within 2 years showed that the area under the curve for $\mathrm{ADC}_{\min }$ was 0.738 (95\% CI, 0.608-0.868; $P=0.001$ ) (Fig. 2). The cutoff value of $\mathrm{ADC}_{\min }$ was $0.482 \times 10^{-3} \mathrm{~mm}^{2} / \mathrm{sec}$, with a sensitivity of $84.3 \%$ and a specificity of $60 \%$. The DFS for patients with $\mathrm{ADC}_{\text {min }} \geq 0.482 \mathrm{~mm}^{2} / \mathrm{sec}$ was significantly higher than that of patients with $\mathrm{ADC}_{\min }<0.482 \mathrm{~mm}^{2}$ / $\sec (P=0.002$, long-rank test $)$ (Fig. 3$)$.

\section{Discussion}

Although CCRT is an optimal therapy for LACC with appreciable outcome, treatment for relapse of tumor afterwards remains tough. Thus we assumed it will be of clinical significance to find high-risk patients who subject to recurrence within short time, and who might benefit from additional or novel therapies, such as targeted agents with chemotherapy [10], or adjuvant or consolidation chemotherapy after CCRT [11]. Therefore, functional MR sequences and imaging processing techniques been investigated for this purpose.

In our study, $\mathrm{ADC}_{\min }$ and $\mathrm{ADC}_{10 \%}$ were significantly different between the recurrence and non-recurrence groups after 2 years of follow-up, while only $\mathrm{ADC}_{\text {min }}$ was found to be correlated with DFS of cervical cancers by 
Table 3 Univariate and multivariate Cox regression analysis of clinical factors and ADC histogram parameters for predicting diseasefree survival

\begin{tabular}{|c|c|c|c|c|}
\hline Parameter & Univariate analysis & $P$-value & Multivariate analysis & $P$-value \\
\hline Age (years) & $1.026(0.987-1.066)$ & $0.194^{*}$ & $1.004(0.963-1.046)$ & 0.858 \\
\hline \multicolumn{5}{|l|}{ Grade } \\
\hline Well/moderate & 1.0 & & & \\
\hline Poorly & $2.731(1.242-6.005)$ & 0.412 & & \\
\hline MTD & $1.004(0.981-1.027)$ & 0.764 & & \\
\hline \multicolumn{5}{|l|}{ FIGO stage } \\
\hline $\mathrm{IB} / \mathrm{IA}$ & 1.0 & & & \\
\hline$\| \mathrm{B} / \mathrm{II} / \mathrm{IV}$ & $1.256(0.572-2.760)$ & 0.570 & & \\
\hline SCC-Ag & $1.004(0.982-1.027)$ & 0.716 & & \\
\hline Median $\left(\times 10^{-3} \mathrm{~mm}^{2} / \mathrm{s}\right)$ & $0.435(0.037-5.075)$ & 0.507 & & \\
\hline $\operatorname{Max}\left(\times 10^{-3} \mathrm{~mm}^{2} / \mathrm{s}\right)$ & $0.918(0.613-1.373)$ & 0.676 & & \\
\hline Mean $\left(\times 10^{-3} \mathrm{~mm}^{2} / \mathrm{s}\right)$ & $0.397(0.032-4.968)$ & 0.474 & & \\
\hline $\operatorname{Min}\left(\times 10^{-3} \mathrm{~mm}^{2} / \mathrm{s}\right)$ & $0.171(0.063-0.463)$ & $0.001 \#$ & $0.110(0.023-0.538)$ & $0.006 \#$ \\
\hline $10 \%\left(\times 10^{-3} \mathrm{~mm}^{2} / \mathrm{s}\right)$ & $0.106(0.006-1.933)$ & $0.130^{*}$ & $1.803(0.069-46.817)$ & 0.723 \\
\hline $90 \%\left(\times 10^{-3} \mathrm{~mm}^{2} / \mathrm{s}\right)$ & $0.793(0.287-2.193)$ & 0.655 & & \\
\hline Kurtosis & $0.950(0.821-0.099)$ & 0.489 & & \\
\hline Skewness & $0.657(0.350-1.233)$ & $0.188^{*}$ & $0.724(0.368-1.463)$ & 0.350 \\
\hline
\end{tabular}

${ }^{*} P<0.3 ; \# P<0.05$

Hazard ratio (HR) data are reported per one-unit increase

Data in parentheses are $95 \%$ confidence intervals
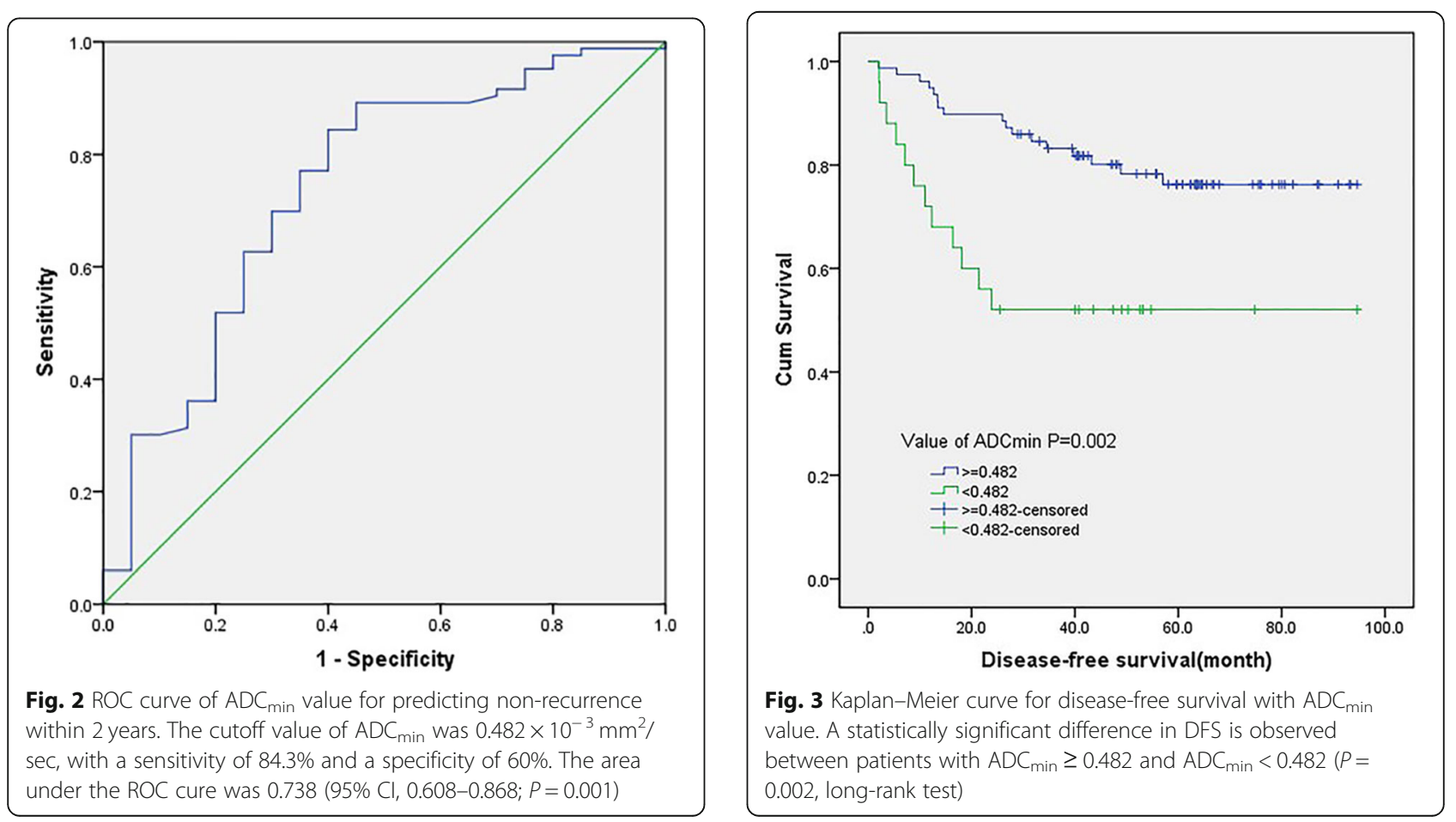
multivariate regression analysis. $\mathrm{ADC}_{10 \%}$ and skewness were not associated with tumor recurrence in the multivariate analysis.

In previous studies, ADC values were found to be lower in cervical squamous cell carcinoma compared with adenocarcinoma, even when using HA-derived mean ADCs [3, 12] and minimal ADCs [13, 14]. Therefore, we only included squamous cell cancers in this study, as it is the most common histologic type of cervical cancer and also to exclude possible interference due to different pathology types. ADC values of malignant tumors are commonly lower than those of normal tissues, as is also the case in uterine cervical cancer $[3,15]$. However, the usefulness of mean ADC values in predicting therapeutic effects differs between studies, possibly due to inter-observer variations in ROI selection and size. The introduction of HA, by further dissecting and defining the distributions of all voxels involved, helps to provide more information for tumor characterization. Theoretically, only ROIs that cover whole lesions are able to comprehensively reflect tumor characteristics. Even necrosis may represent one of the intrinsic features of a tumor, so we think it is not necessary to exclude the possible necrotic areas, as was usually done in previous studies when using small ROIs.

With the method of HA, lower ADC values were often found to be related to more aggressive and poor prognosis tumors, such as poorly differentiated stage I cervical cancers, compared with well/moderately differentiated ones [16]. In a study by Erbay et al. [17], using HA to achieve several percentile $\mathrm{ADC}$ values, they found $\mathrm{ADC}_{50 \%}$, $\mathrm{ADC}_{75 \%}, \mathrm{ADC}_{90 \%}$, and $\mathrm{ADC}_{95 \%}$ were lower in patients with tumor recurrence than in those without recurrence. Unfortunately, the percentiles they used were all above $50 \%$. Similar results were also found in a study by Gladwish et al., in which, among different percentiles of ADC values that were higher than $50 \%$, the $\mathrm{ADC}_{95 \%}$ and $\mathrm{nADC}_{95 \%}$ (normalized to urine) were found to be independent protective factors associated with DFS [18]. Another study showed $\mathrm{ADC}_{75 \%}$ to be a risk factor for tumor recurrence [19]. In our study, high percentiles do not seem to be so significant among all histogram parameters, while the lower $\mathrm{ADC}$ values $\left(\mathrm{ADC}_{\min }\right.$ and $\left.\mathrm{ADC}_{10 \%}\right)$ exhibit a difference between the recurrence and nonrecurrence groups. Several studies have also shown that $\mathrm{ADC}_{10 \%}$ is significantly associated with event-free survival [20] and DFS [21]. However, in our study, low $\mathrm{ADC}_{10 \%}$ values may be correlated with recurrence within 2 years, yet they are not associated with DFS in our Cox regression analysis; $\mathrm{ADC}_{\text {min }}$ is an independent risk factor related to DFS using Cox regression analysis.

Some studies also found that $\mathrm{ADC}_{\text {min }}$ is a valuable factor that reflects tumor aggressiveness. The $\mathrm{ADC}_{\text {min }}$ of stage T2b-T4 cervical cancers was significantly lower than that of stage T1 to T2a cancers, and it was even lower in patients with lymph node metastasis or distant metastasis [22]. In one histopathologic study, the $\mathrm{ADC}_{\text {min }}$ value of cervical cancer exhibited a negative relationship with $\mathrm{Ki}-67$, the index of cellular proliferation $(\mathrm{r}=-0.56, \quad \mathrm{CI}=-0.68--0.43, \quad[23]) . \quad$ A meta-analysis among various kinds of tumors also found $\mathrm{ADC}_{\min }$ had a stronger relationship with Ki-67 compared with $\mathrm{ADC}_{\text {mean }}$ [24]. In a study on malignant astrocytomas, $\mathrm{ADC}_{\text {min }}$ was found to be negatively correlated with the Ki-67 labeling index, which indicates poor prognosis, and the $\mathrm{ADC}_{\text {min }}$ value was found to be significantly higher in the stable group than in the progressing group after treatment [25]. Such results confirm the possible application of $\mathrm{ADC}_{\text {min }}$ in predicting survival rates; higher $\mathrm{ADC}_{\text {min }}$ values may be related to longer DFS, as is also shown in the present study. However, contradictions can also be found in the literature. For example, in a study by Marconi et al. [26], $\mathrm{ADC}_{\min }$ was found to be an independent factor related with DFS as well, but it was a risk factor instead of a protective factor. Considering the fact that HA can also be affected by the coverage extent of ROIs, studies with whole tumor ROIs or single slice ROIs may lead to different results. As stated above, we assumed that whole lesion ROIs can reflect intrinsic tumor heterogeneity to the highest extent, so they are more reliable. The different $b$-values used for DWI scanning form another factor that may affect the results, because there is no single b-value that is uniformly used among studies.

\section{Conclusions}

The volumetric $\mathrm{ADC}_{\min }$ in $\mathrm{HA}$ has the possibility to act as an independent risk factor that is correlated with DFS in cervical cancer patients treated with chemo-radiation therapy. Pre-treatment ADC histogram analysis may identify higher risk of recurrence patients, which will enable greater personalization of cancer care either via a priori treatment decisions or subsequent followup regimens.

\section{Abbreviations \\ ADC: Apparent diffusion coefficient; CCRT: Concurrent chemoradiotherapy; DWI: Diffusion weighted images; FIGO: International Federation of Gynecology and Obstetrics; HA: Histogram analysis; LACC: Locally advanced cervical carcinoma; MDT: Maximal diameter of tumor; NCCN: National Comprehensive Cancer Network; OS: Overall survival; ROI: Regions of interests; ROC: Receiver operating characteristic}

\section{Acknowledgements}

We thank Hui Liu, Ph.D. for integrating and modifying the radiomics software. Also, we thank LetPub (www.letpub.com) for its linguistic assistance during the preparation of this manuscript.

\section{Authors' contributions}

KC and YSS contributed to the conception of the study. XTL performed the data analyses. HTZ contributed significantly to process data. BZ wrote the manuscript. All of the authors read and approved the final manuscript. 


\section{Funding}

This study was funded by Natural Science Foundation of China (grant number 81402167), Beijing Municipal Administration of Hospitals Clinical Medicine Development of Special Funding Support(No.ZYLX201803), Beijing million Talents Project(No.2017A13)and National Key Research and Development Plan(2017YFC1309101, 2017YFC1309104). The funding body had no role in the design of the study and collection, analysis, and interpretation of data and in writing the manuscript.

\section{Availability of data and materials}

The datasets used and analyzed during the current study are available from the corresponding author on reasonable request.

\section{Ethics approval and consent to participate}

The Clinical Research Ethics Committee of Peking University Cancer Hospital and Institute approved the research and the written informed consent was waived because this was a retrospective and observational study.

\section{Consent for publication}

Not applicable.

\section{Competing interests}

The authors declare that they have no competing interests.

Received: 19 July 2019 Accepted: 7 November 2019

Published online: 15 November 2019

\section{References}

1. NCCN Clinical Practice Guidelines in Oncology - Cervical Cancer. In.; 2017.

2. Biewenga P, van der Velden J, Mol BW, Stalpers LJ, Schilthuis MS, van der Steeg JW, et al. Prognostic model for survival in patients with early stage cervical cancer. Cancer. 2011;117(4):768-76.

3. Kuang F, Ren J, Zhong Q, Liyuan F, Huan Y, Chen Z. The value of apparent diffusion coefficient in the assessment of cervical cancer. Eur Radiol. 2013; 23(4):1050-8.

4. Micco M, Vargas HA, Burger IA, Kollmeier MA, Goldman DA, Park KJ, et al. Combined pre-treatment MRI and 18F-FDG PET/CT parameters as prognostic biomarkers in patients with cervical cancer. Eur J Radiol. 2014; 83(7):1169-76.

5. Liu Y, Bai R, Sun H, Liu H, Zhao X, Li Y. Diffusion-weighted imaging in predicting and monitoring the response of uterine cervical cancer to combined chemoradiation. Clin Radiol. 2009:64(11):1067-74.

6. Himoto Y, Fujimoto K, Kido A, Baba T, Tanaka S, Morisawa N, et al. Pretreatment mean apparent diffusion coefficient is significantly correlated with event-free survival in patients with International Federation of Gynecology and Obstetrics Stage Ib to IIIb cervical cancer. Int J Gynecol Cancer. 2015;25(6):1079-85

7. Zhang Y, Chen JY, Xie CM, Mo YX, Liu XW, Liu Y, et al. Diffusion-weighted magnetic resonance imaging for prediction of response of advanced cervical cancer to chemoradiation. J Comput Assist Tomogr. 2011;35(1):102-7.

8. Rizzo S, Summers P, Raimondi S, Belmonte M, Maniglio M, Landoni F, et al Diffusion-weighted MR imaging in assessing cervical tumour response to nonsurgical therapy. Radiol Med. 2011;116(5):766-80

9. Harry VN, Gilbert FJ, Parkin DE. Predicting the response of advanced cervical and ovarian tumors to therapy. Obstet Gynecol Surv. 2009;64(8):548-60.

10. Duenas-Gonzalez A, Zarba JJ, Patel F, Alcedo JC, Beslija S, Casanova L, et al. Phase III, open-label, randomized study comparing concurrent gemcitabine plus cisplatin and radiation followed by adjuvant gemcitabine and cisplatin versus concurrent cisplatin and radiation in patients with stage IIB to IVA carcinoma of the cervix. J Clin Oncol. 2011;29(13):1678-85.

11. Yavas G, Yavas C, Sen E, Oner I, Celik C, Ata O. Adjuvant carboplatin and paclitaxel after concurrent cisplatin and radiotherapy in patients with locally advanced cervical cancer. Int J Gynecol Cancer. 2019;29(1):42-7.

12. Guan Y, Shi H, Chen Y, Liu S, Li W, Jiang Z, et al. Whole-lesion histogram analysis of apparent diffusion coefficient for the assessment of cervical cancer. J Comput Assist Tomogr. 2016:40(2):212-7.

13. Xue H, Ren C, Yang J, Sun Z, Li S, Jin Z, et al. Histogram analysis of apparent diffusion coefficient for the assessment of local aggressiveness of cervical cancer. Arch Gynecol Obstet. 2014;290(2):341-8.
14. Liu Y, Ye Z, Sun H, Bai R. Clinical application of diffusion-weighted magnetic resonance imaging in uterine cervical cancer. Int J Gynecol Cancer. 2015; 25(6):1073-8.

15. Chen J, Zhang Y, Liang B, Yang Z. The utility of diffusion-weighted MR imaging in cervical cancer. Eur J Radiol. 2010;74(3):e101-6.

16. Downey K, Riches SF, Morgan VA, Giles SL, Attygalle AD, Ind TE, et al. Relationship between imaging biomarkers of stage I cervical cancer and poor-prognosis histologic features: quantitative histogram analysis of diffusion-weighted MR images. AJR Am J Roentgenol. 2013;200(2):314-20.

17. Erbay G, Onal C, Karadeli E, Guler OC, Arica S, Koc Z. Predicting tumor recurrence in patients with cervical carcinoma treated with definitive chemoradiotherapy: value of quantitative histogram analysis on diffusionweighted MR images. Acta Radiol. 2017;58(4):481-8.

18. Gladwish A, Milosevic M, Fyles A, Xie J, Halankar J, Metser U, et al. Association of Apparent Diffusion Coefficient with disease recurrence in patients with locally advanced cervical cancer treated with radical chemotherapy and radiation therapy. Radiology. 2016;279(1):158-66.

19. Heo SH, Shin SS, Kim JW, Lim HS, Jeong YY, Kang WD, et al. Pre-treatment diffusion-weighted MR imaging for predicting tumor recurrence in uterine cervical cancer treated with concurrent chemoradiation: value of histogram analysis of apparent diffusion coefficients. Korean J Radiol. 2013;14(4):616-25.

20. Ueno Y, Lisbona R, Tamada T, Alaref A, Sugimura K, Reinhold C. Comparison of FDG PET metabolic tumour volume versus ADC histogram: prognostic value of tumour treatment response and survival in patients with locally advanced uterine cervical cancer. Br J Radiol. 2017;90(1075):20170035.

21. Lin G, Yang LY, Lin YC, Huang YT, Liu FY, Wang CC, et al. Prognostic model based on magnetic resonance imaging, whole-tumour apparent diffusion coefficient values and HPV genotyping for stage IB-IV cervical cancer patients following chemoradiotherapy. Eur Radiol. 2019;29(2):556-65.

22. Grueneisen J, Schaarschmidt BM, Heubner M, Aktas B, Kinner S, Forsting M, et al. Integrated PET/MRI for whole-body staging of patients with primary cervical cancer: preliminary results. Eur J Nucl Med Mol Imaging. 2015; 42(12):1814-24.

23. Surov A, Meyer HJ, Wienke A. Associations between apparent diffusion coefficient (ADC) and KI 67 in different tumors: a meta-analysis. Part 2: ADCmin. Oncotarget. 2018;9(9):8675-80.

24. Surov A, Meyer HJ, Schob S, Hohn AK, Bremicker K, Exner M, et al. Parameters of simultaneous 18F-FDG-PET/MRI predict tumor stage and several histopathological features in uterine cervical cancer. Oncotarget. 2017:8(17):28285-96

25. Higano S, Yun X, Kumabe T, Watanabe M, Mugikura S, Umetsu A, et al. Malignant astrocytic tumors: clinical importance of apparent diffusion coefficient in prediction of grade and prognosis. Radiology. 2006;241(3): 839-46.

26. Marconi DG, Fregnani JH, Rossini RR, Netto AK, Lucchesi FR, Tsunoda AT, et al. Pre-treatment MRI minimum apparent diffusion coefficient value is a potential prognostic imaging biomarker in cervical cancer patients treated with definitive chemoradiation. BMC Cancer. 2016;16:556.

\section{Publisher's Note}

Springer Nature remains neutral with regard to jurisdictional claims in published maps and institutional affiliations.

Ready to submit your research? Choose BMC and benefit from:

- fast, convenient online submission

- thorough peer review by experienced researchers in your field

- rapid publication on acceptance

- support for research data, including large and complex data types

- gold Open Access which fosters wider collaboration and increased citations

- maximum visibility for your research: over $100 \mathrm{M}$ website views per year

At $\mathrm{BMC}$, research is always in progress.

Learn more biomedcentral.com/submission 